\title{
Photonic logic based on molecular reorientation of nematic liquid crystals
}

\author{
By J. A. Martin-Pereda, F. J. López, M. A. Muriel and J. M. Otón \\ Departamento Electronica Cuantica, E.T.S. Ing. Telecommunicacion U.P.M., \\ Ciudad Universitaria, 28040-Madrid, Spain
}

\begin{abstract}
A new type of photonic logic, based on the use of nematic liquid crystals is proposed. The system takes advantage of the refractive-index changes induced by laser beams. Examples of AND, OR and NOR functions are presented.
\end{abstract}

It is nowadays a well-known fact that nematic liquid crystals offer a very interesting set of large optical nonlinearities. As has been shown, both theoretically (Khoo I98I) and experimentally (Khoo I980, I982), these nonlinearities come from the optical field-induced, collective reorientation of the nematic molecules. A continuous wave (c.w.) laser of moderate power can be intense enough to yield a significant refractive index change in the medium. Moreover, this change has an appreciable effect on laser beam propagation and, hence, output laser beam characteristics can be very different from the input ones. One of the more promising effects to be applied in optically bistable devices is the one reported by Zolot'ko et al. (1980) and by Durbin et al. (198I). They have reported the observation of a multiple-ring pattern of laser diffraction from a nematic liquid crystal film. This phenomenon appears for a certain laser intensity level and its time responses depend on both cell thickness and molecular orientation, the configuracion employed most often being the homeotropic one. If the laser power is below the threshold, no diffraction phenomenon is obtained and both laser beams, namely input and output beams, have similar characteristics. Moreover, as we have shown Martín-Pereda ( 1982 , 1983), if two laser beams cross the cell at the same point, one beam being more intense than the other, the output characteristics of the smaller one can be affected by the larger one. This effect has been reported as opto-optical modulation, with possible applications in all-optical devices.

The above facts have been employed to develop optical logic gates; AND, OR, NOR and NAND functions have been achieved. The basis of the operation is the ability of a nematic homeotropic cell to alter its molecular orientation according to the crossing total laser intensity regardless of whether this intensity comes just from one laser beam or from more than one. A cooperative effect is the result.

Planar $120 \mu \mathrm{m}$ thick structures were used.' The homeotropic alignment was obtained by surface treatment with hexadecyltrimethyl ammonium bromide (HTAB); p-methoxybenzylidene- $\mathrm{p}^{\prime}$-n-bulylaniline (MBBA) was employed as nematic liquid crystal, and the experiments were made at room temperature (about $\left.25^{\circ} \mathrm{C}\right)$, i.e. within the nematic range of MBBA.

The experiments were made with c.w. $\mathrm{Ar}^{+}$ion lasers working at their green line $(514.5 \mathrm{~nm})$. The optical propagation was, in every case, at an angle of $0^{\circ}$ with the liquid crystal molecular axis.

The system shown in figure 1 gives, as will be shown, AND and OR logical functions. LG $_{1}$ 


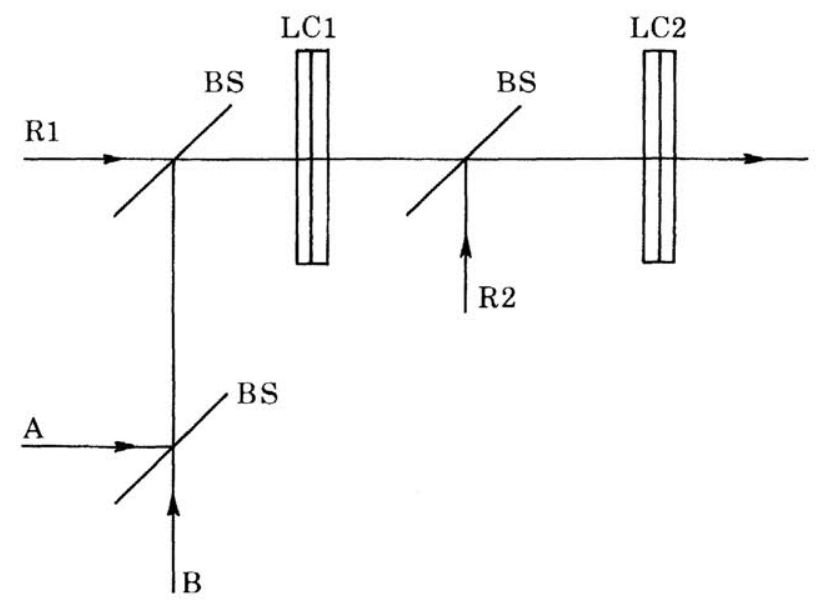

Figure 1. A system for AND and OR logical functions.

and $\mathrm{LC}_{2}$ are two homeotropic liquid crystal cells. BS are beam splitters and $R_{1}$ and $R_{2}$ two read-out laser beams. A and B are the two possible inputs. The behaviour of the system is now described.

\section{AND FUNGTION}

If $A$ and $B$ inputs are non-zero, the light crossing $L C_{1}$ has a level corresponding to the addition of $A, B$ and $R_{1}$. Under these circumstances, the system is adjusted in such a way that the total power impinging on the nematic cell is above the threshold. Hence, the output from $\mathrm{LC}_{1}$ is diffraction rings. The light crossing $L_{2}$ is just $R_{2}$ because no appreciable contribution comes from the exit of $L C_{1}$. If the light intensity of $R_{2}$ is below the $L_{2}$ threshold, the output of the system will be $R_{2}$. So, the system output corresponds to one.

If either $A$ or $B$ are zero, the light crossing $L_{1}$ is $R_{1}+A$ (or $B$ ). If it is below the $L_{1}$ threshold, the light going onto $L_{2}$ will be $R_{2}+R_{1}+A$ (or $R_{2}+R_{1}+B$ ). Because this power level is now over the $\mathrm{LC}_{2}$ threshold, the system output is zero.

If both $A$ and $B$ are zero, the light crossing $L_{2}$ is $_{1}+R_{2}$. And because the system is adjusted in such a way that this intensity is over the threshold level, the system output will be zero.

\section{OR FUNGTION}

The system works in a similar way to that described for the AND function. The only difference is the threshold level of the $\mathrm{LC}_{1}$ cell. In this case, just when the crossing light is $R_{1}$, the power is below the threshold. When $\mathrm{A}$ or $\mathrm{B}$ are different from zero, $\mathrm{LC}_{\mathbf{1}}$ will form diffraction rings and the system output will be 1 .

\section{NOR FUNGTION}

A system like that shown in figure 2 is able to work as a NOR function. The behaviour is similar to that described for AND and OR functions. The difference is that just one read-out laser beam is needed. When the light crossing $L C$ is just $R_{1}$, no diffraction rings appear 


\section{PHOTONIC LOGIC BY USING LIQUID GRYSTALS}

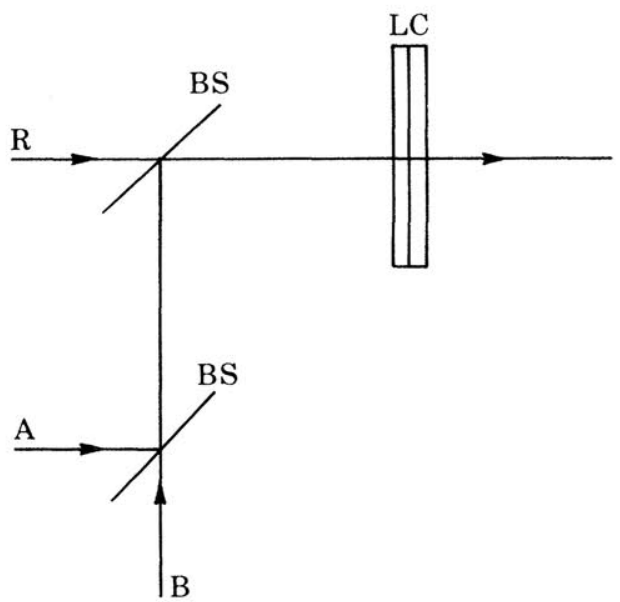

Figure 2. A system for a NOR function.

because it is below the threshold. Hence the system output will be 1 . But when any one of A or B (or A and B) are present the light intensity level is over the threshold so that a zero is obtained. The advantage of this system is that, as is well known from logic, with this function any other function can be achieved.

To get some feeling of the intensity levels required from each of the three working laser beams some calculations need to be done. If we arbitrarily assign 100 to the power level needed for diffraction rings to arise the following conditions are obtained:

$$
\begin{gathered}
\frac{1}{4} \mathrm{~A}+\frac{1}{2} \mathrm{R}>100 ; \\
\frac{1}{2} \mathrm{R}<100 ; \quad \frac{1}{4} \mathrm{~A}<100 ; \\
\frac{1}{2} \mathrm{R}>\mathrm{A} .
\end{gathered}
$$

The first condition comes from the fact that $\mathrm{A}+\mathrm{B}$ must give 0 . The second one is imposed by the need to have no diffraction rings when any one of them is working alone. The last one is obtained from the fundamental requirement for any computer that the output of a gate be strong enough to drive other gates. As can be seen, the major drawback of the system comes from the beam splitters, because a lot of optical power is lost at them.

With these conditions a diagram, like the one shown in figure 3, can be drawn. As can be seen, there is just one region where the system can work. The numbers express the power needed by each beam, 100 being the threshold level for the appearance of diffraction rings at the liquid crystal cell output. The beam splitters have been designed in such a way that $50 \%$ of the input power is reflected and $\mathbf{5 0} \%$ transmitted. Hence, the laser intensity crossing the cell is given by

$$
I_{\mathrm{LC}}=\frac{1}{2} \mathrm{R}+\frac{1}{4} \mathrm{~A}+\frac{1}{4} \mathrm{~B} \text {. }
$$

If some other beam-splitter characteristics are assumed, this relation must be modified.

According to our previous work, $\mathrm{Ar}^{+}$intensities of about $3 \mathrm{~kW} \mathrm{~cm}$ che $^{-2}$ are needed for the rings to be formed. The ranges of intensities of laser beams $A, B$ and $R$ are

$$
\begin{gathered}
4.5 \mathrm{~kW} \mathrm{~cm}^{-2}<\mathrm{R}<6 \mathrm{~kW} \mathrm{~cm}^{-2}, \\
\mathrm{~A}, \mathrm{~B}<3 \mathrm{~kW} \mathrm{~cm}{ }^{-2} .
\end{gathered}
$$

These values are in very good agreement with our experimental values. 


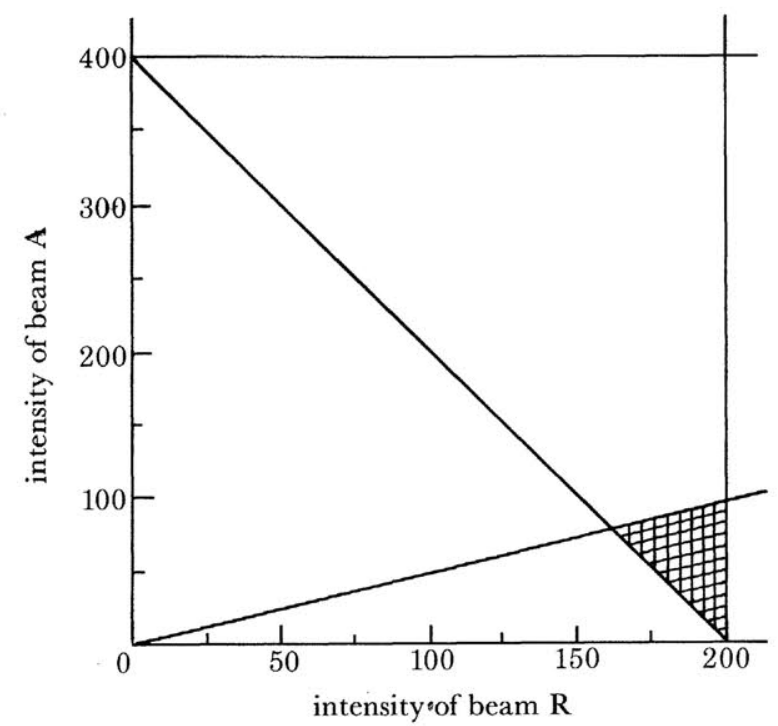

Figure 3. Diagram to indicate the working zone (hatched area).

An advantage of this type of logic gate is that its working conditions are almost absolutely independent of the acting wavelengths. In fact, we have also made our experiments with two types of lasers, namely an $\mathrm{Ar}^{+}$laser as a read-out laser and a $\mathrm{He}-\mathrm{Ne}$ laser for data entry. This allows the possibility of a clear separation between both types of beams.

This work was supported by the Spanish Comision Asesora de Investigación Científica y Técnica Grant no. 1564-82.

\section{REFERENGES}

Durbin, S. D., Arakelian, S. M. \& Shen, Y. R. r981 Optics Lett. 6, 411.

Khoo, I. C. 1982 Phys. Rev. A25, 1040.

Khoo, I. C. \& Zhuang, S. L. 1980 Appl. Phys. Lett. 37, 3.

Khoo, I. C., Zhuang, S. L. \& Shepard, S. 1981 Appl. Phys. Lett. 39, 937.

Martín-Pereda, J. A. \& López, F. J. 1982 Optics Lett. 7, 590.

Martín-Pereda, J. A., Lopez, F. J. \& Muriel, M. A. 1983 Molec. Cryst. liq. Cryst. 99, 1.

Zolot'ko, A. S., Kitaeva, V. F., Sobolov, N. N. \& Csillag, L. I980 Soviet Phys. JETP Lett. 32, 158. 